\title{
editorial
}

\section{El contrato de gestión en los centros de Atención Primaria}

\begin{abstract}
$\mathscr{L}$ búsqueda de instrumentos que mejoren la eficiencia del sistema sanitario y faciliten la consecución de sus objetivos, ha provocado distintos cambios en la gestión, uno de ellos, que se viene utilizando desde hace varios años, es el esquema del Contrato de Gestión (CG). Esta figura se inicia a partir del año 1992 a raíz de la publicación del Real Decreto $858 / 1992^{1}$ que define la organización y competencias del Ministerio de Sanidad y Consumo (MSyC) y las propias del INSALUD, en un intento de separar las funciones de planificación, financiación, compra y provisión ${ }^{2,3}$, de los servicios sanitarios.

La separación de funciones ha sido uno de los motores de la reforma en distintos países de nuestro entorno socioeconómico, fue una de las principales recomendaciones de la Comisión de Análisis, Evaluación y Reforma del Sistema Nacional de Salud (SNS) - Informe Abril - y uno de los puntos del consenso en el Parlamento reflejado en el sumario final de la Subcomisión de Consolidación y Modernización de $\mathrm{SNS}^{4}$, que proponía proseguir en los esfuerzos por diferenciar las funciones y configurar los centros asistenciales como organizaciones autónomas, con facultades de decisión efectivas y responsables en cuanto a la gestión.

Para describir de forma resumida la responsabilidad de cada uno de estos agentes ${ }^{5}$, diríamos que la función de autoridad (financiación) recae en el MSyC, al que le corresponden los aspectos de elaboración del Plan de Salud para orientar todas las acciones sanitarias, determinar las prestaciones en función de las prioridades establecidas por el Plan y las disponibilidades financieras y establecer los recursos económicos necesarios para hacer efectivas las prestaciones de una forma equitativa a toda la población.

La función de aseguramiento (compra) recae en los Servicios Regionales de Salud de las CCAA o en el INSALUD en aquel territorio no transferido aún. Sus actividades más importantes son las de adecuar la oferta de servicios a las necesidades de cada territorio, establecer los criterios de distribución territorial de presupuestos y objetivos y establecer la política de compra de prestaciones sanitarias con los centros proveedores o de las prestaciones complementarias (transporte sanitario, orto-prótesis, etc.).

Por último la función de provisión recae en los centros sanitarios con la responsabilidad de ofertar unos servicios de calidad a la población, accesibles y satisfactorios, que estarían dotados de la autonomía e incentivos suficientes para cumplir los objetivos dentro del presupuesto asignado.
\end{abstract}


En este esquema de división funcional, la figura del CG se constituye como un instrumento de gestión de los centros sanitarios públicos que vincula el presupuesto con la actividad. Las prioridades de planificación, originadas por el conocimiento de las necesidades de salud y la disponibilidad de medios, se trasladan hacia el plan de gestión del INSALUD, que decodifica estas decisiones de planificación en términos de objetivos asistenciales y los transmite a las Gerencias de Atención Primaria y Atención Especializada por medio de sus respectivos CG.

Las Gerencias reparten entre las unidades y centros sus correspondientes objetivos, quedando constituidos los Contratos de Gestión Clínico-Asistencial (CGCA), cuya suma contempla la globalidad de las acciones y recursos definidos en el CG del Área.

La aplicación habitual de los CG en Atención Primaria, implica la negociación y pacto con cada uno de los Centros de Salud para fijar de manera concreta los aspectos de distintos apartados y que habitualmente contemplan:

- La definición de objetivos de organización, Cartera de Servicios, calidad, satisfacción de usuarios, uso racional del medicamento e incapacidad temporal.

- La asignación de los recursos definidos tanto en términos económicos y clasificados en sus distintos apartados presupuestarios, como otros recursos asistenciales de interconsultas con atención especializada y pruebas diagnósticas.

- La relación de las decisiones que se descentralizan y que suelen reconocer competencias de organización y funcionamiento del centro, la autorización de licencias y permisos, la selección de personal eventual y la gestión de los presupuestos asignados.

- La bolsa de incentivos a recibir en el caso de correcto cumplimiento del CGCA.

Como es lógico, este procedimiento pretende mejorar la eficiencia técnica, contener la inflación presupuestaria, crear un ambiente más competitivo y favorecer la descentralización en la toma de decisiones, permitiendo incluso la utilización del excedente económico que se pueda generar por las medidas de buena gestión, en inversiones para mejorar el funcionamiento o en distintas alternativas de motivación profesional (docencia, investigación, incentivos económicos, ...).

Después de estos años de funcionamiento podríamos realizar algunos comentarios sobre sus ventajas e inconvenientes:

-Una de las mayores críticas se sitúan en su carácter de ficción legal donde sigue pesando excesivamente el esquema jerárquico, la ausencia de riesgo y responsabilidad si no se alcanzan los compromisos pactados. Este sometimiento a la autoridad resta autonomía a cada una de las partes que se sientan en la negociación, bien sean en el cierre del acuerdo del CG del Área o de los CGA de los Centros'.

Aunque la publicación de distintas normas legislativas sobre nuevas 
fórmulas de gestión, ha posibilitado la sustitución de este procedimiento de "pacto entre caballeros" por una relación contratractual de carácter más formal y la constitución real de centros autogestionados, por distintos motivos no ha llegado a materializarse el reemplazo necesario.

-Existe una escasa asociación con los objetivos definidos en los Planes de $\mathrm{Salud}^{7}$, tanto el del MSC como el de los determinados para cada uno de los distintos territorios autonómicos. Los objetivos asistenciales están fundamentalmente basados en un incremento de actividad y cobertura de la población por los distintos servicios, en un intento de mejorar el rendimiento de la capacidad instalada, que suele ser meritorio pero a veces no pertinente o sin repercusiones positivas sobre el estado de salud.

Hay una actualización permanente de la estructura y contenido de la Cartera de Servicios que la adaptan a los nuevos problemas de salud que van surgiendo, sin embargo esta transformación es común y uniforme para todo el territorio del INSALUD y está poco vinculada con las prioridades que marcan los documentos de análisis de necesidad.

Esta situación de distanciamiento entre la necesidad y la actividad, desvirtúa los fundamentos previstos para los CG y desaprovecha la oportunidad que posee el financiador para estimular la realización de aquellas acciones sanitarias que marcan las prioridades del SNS, situación que además es agravada por la separación y aislamiento de la red asistencial con la de salud pública.

- La actividad y el presupuesto poseen poca relación y la financiación está basada en criterios históricos de presupuesto y en la ejecución del gasto de ejercicios anteriores. En el caso de Atención Primaria el presupuesto debería estar asociado a la población cubierta ponderada por los factores sociosanitarios que modifican la actividad asistencial. El peso del componente histórico está motivado por la gran dificultad que existe para redistribuir los recursos desde los territorios geográficos (áreas de salud o zonas básicas) que van perdiendo población, bien sea esta disminución asociada a los movimientos demográficos o por que los ciudadanos eligen otros centros o profesionales que les ofrecen mejor accesibilidad o calidad en la atención.

Dado que existe experiencia en incorporar criterios de población ponderada en la asignación del presupuesto de prescripción farmacéutica se podría extender este método, aunque utilizando otras variables que permitan una asignación más ajustada, hacia el resto de los capítulos presupuestarios y avanzar hacia un presupuesto prospectivo por capitación.

- Ha mejorado la descentralización y el nivel de autonomía de los centros. El beneficio teórico esperado por la devolución de la toma de decisiones sobre la organización de su trabajo a los centros y profesionales ${ }^{8}$, está basado en las ventajas que aporta en la utilización de la información local y permitir una mayor adaptación de los servicios a situacio- 
nes particulares, un incremento de la productividad, una disminución de los costes así como un perfeccionamiento del servicio con el incremento de satisfacción de usuarios y profesionales. Estas ventajas teóricas están teniendo una correlación con la evolución temporal de los indicadores de evaluación, si bien es imposible atribuirlas exclusivamente al uso de CG.

-El seguimiento longitudinal de la gestión presupuestaria de los centros y la comparación entre centros de distintas Comunidades Autónomas que trabajaban con y $\sin \mathrm{CG}^{9}$, resalta que aunque los objetivos de contención de gasto eran excesivamente optimistas, sí se ha conseguido una mayor disciplina financiera y un incremento significativo de la eficiencia relativa, lo que sin duda tiene efectos beneficiosos tanto para la contención del endeudamiento público como para la ganancia de credibilidad del sector sanitario en la gestión de los recursos que los ciudadanos depositan en él.

-Por último, y probablemente el efecto más importante, es el cambio en el método de trabajo y cultural que ha supuesto el CG, gracias a la introducción de una dirección participativa por objetivos. La necesidad de hacer el ejercicio anual entre los distintos niveles de la organización, de preparar los objetivos e iniciar el circuito de propuesta de objetivos - negociación - pacto - evaluación y nuevo proceso de propuesta para el periodo próximo, está permitiendo desarrollar una mayor comunicación y conocimiento interno, han facilitado la mejora y fiabilidad de la información y sentado un procedimiento estable y periódico de evaluación. En organizaciones donde la gestión del conocimiento es tan importante como es en la nuestra, el poseer instrumentos que la faciliten, mantiene una importancia estratégica.

Existen también críticas hacia la totalidad del instrumento del CG como de las líneas asociadas a él, cuestionando la utilidad de definir objetivos, la existencia de incentivos en los servicios públicos o la pertinencia de los procedimientos de evaluación o los indicadores utilizados; desde nuestra opinión los que mayor fundamento poseen son los que proponen utilizar la resolución de los procesos clínicos como indicadores de efectividad y su asociación a los recursos empleados para poder comparar la eficiencia. La aparición de herramientas informáticas de gestión de la historia clínica está permitiendo caminar hacia esta dirección y ya van apareciendo los primeros resultados.

Como recomendaciones finales, además de las ya comentadas con anterioridad, nos atreveríamos sugerir:

- Realizar un intento que introduzca la orientación de los CG desde la Salud Pública y los Planes de salud. Podría realizarse por medio de la incorporación de un número reducido de objetivos, nunca más de cinco, que marcarán la prioridad de la autoridad sanitaria y que tuvieran un plazo de ejecución razonable, de forma que su consecución produjera ganancias en el nivel de salud e incrementara la legitimidad de nuestros servicios ante la sociedad. 
También se podría acrecentar este vínculo entre la salud pública y la Atención Primaria por medio de la incorporación parcial de funciones y dispositivos salubristas en la Gerencias de AP, que facilitara el diseño de servicios, pacto de actividades y objetivos desde el conocimiento real de la epidemiología y morbilidad local de cada Área ${ }^{10}$.

-Facilitar una gestión global e integral del presupuesto que permita la sustitución e intercambio de los medios productivos y permita mayor agilidad en la gestión económica. Mientras se mantengan las dificultades para confeccionar un presupuesto prospectivo, donde el dinero siga al paciente, sería recomendable que transitoriamente las Gerencias tuvieran algo que ganar o perder y la introducción de modelos de competición por torneo podrían incrementar la eficiencia, sin que esto suponga un abandono de la necesidad avanzar en la asignación de un presupuesto capitativo.

- Aumentar el plazo de vigencia del CG, que en la actualidad es anual, para aportar un plazo temporal mayor que permita la consecución de los objetivos de salud y la modificación del comportamiento de los proveedores.

Es posible que el proceso de descentralización de la gestión sanitaria a la Comunidades Autónomas, haga que las recomendaciones últimas sean baldías y que en los próximos meses presenciemos la desaparición de los CG y su sustitución por otro instrumento asociado a una nítida separación entre los agentes.

La constitución de distintas entidades con gestión autónoma va a eliminar muchas de las actuales limitaciones de los CG, sin embargo no debemos desaprovechar el conocimiento aportado por esta experiencia, ya que siempre existirán unidades clínico-asistenciales de un tamaño menor (salvo que vayamos, la inteligencia no lo permita, a la práctica solitaria del médico de familia) que no posean autonomía propia de gestión y que se verán beneficiadas por el uso de un instrumento, que con independencia de su nombre, les dote de una descentralización delegada y les vincule la actividad a realizar con el presupuesto en un decidido avance hacia la gestión clínica.

L. MORELL

\section{BIBLIOGRAFÍA}

1. Real Decreto-Ley 858/ 1992 de 10 de Julio por el que se define la estructura básica del Ministerio de Sanidad y Consumo. BOE Jueves 16 de julio de 1992; 170: 24606- 12.

2. Ibern P. Las funciones de financiación, compra y provisión de servicios en los sistemas nacionales de salud. Jano 1991; XLI (965): 55- 65.

3. López-Casasnovas G. Descentralización y simulación de mercados: instrumentos para la eficiencia en el sector sanitario público. Papeles de Economía Española 1989; 1: 172-80. 
4. Consolidación y Modernización del Sistema Nacional de Salud (Acuerdo parlamentario de 18 de Diciembre de 1997). Madrid: Secretaría General Técnica. Centro de Publicaciones. Ministerio de Sanidad y Consumo 1998; 35198-003- 7.

5. Elola J. Sistema Nacional de Salud: evaluación de su eficiencia y alternativas de reforma. Barcelona: SG Editores 1994; 171-6.

6. Martín J, Cabasés J. Cambios en la Gestión Sanitaria. En: Navarro C, Cabasés J, Tormo MJ. La salud y el Sistema Sanitario en España. Informe Sespas 1995. Barcelona: SG Editores SA 1995; 203-11.

7. Cabasés J, Gaminde I, Gabilondo L. Contracting arrangemets in the health strategy contex. A regional approach for Spain. European J Pub Health 2000; 10 (4 suppl): 45-50.

8. Matesanz R. Gestión Clínica: ¿por qué y para qué?. Med Clin 2001; 117: 222-6.

9. López-Valcarcel B, Pellisé L, Barber P. La financiación Pública de los servicios sanitarios en España. Documento de Trabajo 95-13. Madrid: Fundación de Estudios de Empresa Aplicada FEDEA 1995.

10. Junta Directiva de AMAS. Los instrumentos de gestión horizontal para gestionar y aprovechar la inter-dependencia. En: Servicio Madrileño de salud: reflexiones y propuestas de futuro. Madrid: Asociación Madrileña de Administración Sanitaria (AMAS) 2000; 14-8. 